\title{
Systematic Errors in Double Star Observations
}

\author{
GEOFFREY G. DOUGLASS \& CHARLES E. WORLEY \\ U.S. Naval Observatory, Washington, DC 20392, USA
}

\begin{abstract}
We have examined the visual and speckle observations of visual double stars made by 31 experienced observers in order to evaluate possible systematic errors in these series. Using 57 "definitive" orbits, we find no appreciable errors in position angle. We do find systematic effects in separation for some observers, and, in particular, a "proximity" effect for measurements of pairs closer than 0 ".5.
\end{abstract}

Root-mean-square residuals are:

\begin{tabular}{|lcc|}
\hline & position angle & separation \\
\hline Visual (telescopes $<41 \mathrm{~cm})$ & $0^{\prime \prime} .05$ & $0^{\prime \prime} .09$ \\
Visual (telescopes $>41 \mathrm{~cm})$ & 0.04 & 0.07 \\
Speckle (2-4 m telescopes) & 0.02 & 0.02 \\
\hline
\end{tabular}

\section{INTRODUCTION}

We have examined 8970 visual double-star means (31665 observations) from the WDS (Washington Double Star) Catalog made by 30 experienced visual observers, together with 617 means obtained by speckle interferometry (CHARA), in order to evaluate possible systematic errors present in these series. The range of dates of observation are 1820 to the present. The experienced visual observers chosen were Aitken, Baize, van den Bos, Bowyer, Bryant, Burnham, Comstock, Couteau, Dembowski, Doberck, Doolittle, Duruy, Finsen, Hall, Heintz, Holden, Hussey, Leavenworth, Lewis, Madler, Muller, Phillips, Rabe, Schiaparelli, F. Struve, H. Struve, O. Struve, van Biesbroeck, Voute, and Worley. For the 57 double-star orbits that are considered "definitive" (Worley \& Heintz 1983, and later orbits), residuals were computed in position angle and separation. To be classed as "definitive" a double star orbit must have well-distributed coverage, observations for more than one revolution, and no revisions to the orbit expected except for minor adjustments.

Earlier we reported on a similar study for eleven visual observers using 51 "definitive" orbits (Worley \& Douglass 1970). Since many of the orbits lacked sufficient observational data, especially for the period prior to 1900 , we did not publish our results. Since then, a significant fraction of the orbits have been improved, and data for most of the pre- 1900 observers has been added to the WDS database by Worley. A study reaching similar conclusions, but based on considerably less data, has also been made by Pannunzio, et al. $(1986,1988)$ for most of the experienced visual observers.

Residuals in position angle and separation for the date of observation were calculated for each orbit. The position angle was precessed to the equinox of the orbit if such was listed. Otherwise, no precession was applied. The apparent orbit, and orbital residuals, were plotted in order to determine the quality of the orbital data. Residuals exceeding 3.5 sigma in either position angle or separation were eliminated, as were observations listed as uncertain by the observer, or if 
either position angle or separation were not measured for a given date. Twentyone of the stars had multiple orbits. For each of these stars the orbit exhibiting the least scatter and smallest residuals was selected (not necessarily the latest orbit). New orbits were computed for STF 1196 ( $\zeta$ Cnc; Harrington, 1992) and STF 1523 ( $\xi$ UMa; Harrington, 1991) in order to account for the effects of the third component of these systems, and for BU 1270 (Douglass, 1991) in which the latest position angle observations no longer agreed with the computed orbit. The final orbital residuals were grouped by observer, and by telescope aperture (less than $41 \mathrm{~cm}$, greater than $41 \mathrm{~cm}$ ).

\section{RESULTS}

The dependence of the residuals in position angle and separation versus time (epoch of observation) was tested. No evidence of any sizeable systematic effects in position angle were found, although several observers showed moderate scatter $\left( \pm 0^{\prime \prime} .1\right)$. There was evidence of systematic effects in separation for some observers (Burnham, Dembowski, Doolittle, Finsen with micrometer, Heintz, O. Struve, van Biesbroeck for apertures less than $41 \mathrm{~cm}$ ). Most observers showed much larger scatter in separation compared to that for position angle, as was expected.

The dependence of the residuals in position angle, and separation, versus observed separation was also tested. Systematic effects appeared in the measures of several observers in position angle (Baize, Rabe, Schiaparelli, F. Struve - all for apertures less than $41 \mathrm{~cm}$; Hall for apertures greater than $41 \mathrm{~cm}$ ), and separation (Dembowski, Doberck, Leavenworth, F. Struve, van Biesbroeck, Voute - all for apertures less than $41 \mathrm{~cm}$; Bryant, Burnham, Doolittle, Heintz, Hall, Lewis, H. Struve - all for apertures greater than $41 \mathrm{~cm}$ ).

A "proximity" effect exhibited by some observers when measuring pairs with separations less than 0 ".5 was detected (Aitken, Bowyer, Bryant, Burnham, Comstock, Doolittle, Duruy, Hall, Hussey, Lewis, Leavenworth, Madler, Rabe, Schiaparelli, F. Struve, H. Struve, O. Struve, and van Biesbroeck and Voute only for apertures less than $41 \mathrm{~cm}$ ).

Scatter and systematic effects for position angle data was very small compared to that in separation. This supports the contention that position angle can be more accurately measured than separation. Surprisingly, the residuals for Heintz for large telescope apertures (greater than $41 \mathrm{~cm}$ ) are slightly worse in position angle than in separation. For all the observers there is larger scatter in the residuals for the smaller (less than $41 \mathrm{~cm}$ ) telescopes. Some of the experienced observers had very few observations of these definitive orbit stars (Aitken, Burnham, Heintz, Hussey, Madler, and H. Struve - all for telescopes less than $41 \mathrm{~cm}$; Leavenworth for telescopes larger than $41 \mathrm{~cm}$ ). Therefore, it was not possible to determine the existence of any systematic effects in those observations. The three Greenwich observers (Bowyer, Bryant, and Lewis) exhibit very large scatter in all their measurements probably due to a combination of poor telescope mounting, optics, and observing climate. The residuals in separation versus separation and also date for Dembowski exhibit very large scatter. This is probably a result of poor measuring equipment. Hall exhibits unusually large scatter in the residuals for separation for measurements made with a large telescope. 
The visual interferometry done by Finsen displays very small scatter in both position angle and separation, although larger than speckle interferometry. There is possibly a slight proximity effect $\left(+0^{\prime \prime} .01\right)$ for the very closest separations. The speckle interferometry measures show the expected extremely small scatter, no evidence of systematic errors, and no "proximity" effect.

For two of the orbits, the speckle interferometry was directly compared with the visual measures of Aitken (A 88), and the visual interferometry measures of Finsen (FIN 312). As expected, both observers show some scatter, and systematic effects compared with speckle interferometry. Separating actual systematic differences from the errors in orbital elements for these two stars appears impossible.

For the visual observations made with smaller (less than $41 \mathrm{~cm}$ ) telescopes, the root-mean-square residuals in position angle and separation are 0.05 and $0 " .09$, respectively. For large (greater than $41 \mathrm{~cm}$ ) telescopes, it is $0^{\prime \prime} .04$ and $0^{\prime \prime} .07$. For speckle interferometry it is $0^{\prime \prime} .02$ and $0 \prime .02$ for all orbits; $0^{\prime \prime} .004$ and 0 "..005 for speckle orbits only. As would be expected, the residuals decrease with increasing telescope aperture.

\section{CONCLUSION}

Except as noted above, there is no evidence for the existence of serious systematic errors in visual, visual interferometry, or speckle interferometry double-star measures made by experienced observers even with small telescopes. We contend that the observed discrepancy in the "sun-Sirius" versus Hyades massluminosity relations cannot be due to alleged systematic errors in the visual measures of the double-star separations.

Speckle observations should be weighted much more heavily in orbit calculations than visual observations, due to their extremely small residuals and the "impersonal" nature of the measurements. Unfortunately, many orbit computers fail to do so.

An expanded version of this study, including tables and figures, will be published at a later date.

\section{REFERENCES}

Douglass, G.G. 1991, unpublished

Harrington, R.S. 1991, unpublished

Harrington, R.S. 1992, IAU Colloquium No. 135

Pannunsio, R., Zappala, V. ,Massone. G., \& Morbidelli, R. 1986, $A \& A, 166,337$

Pannunsio, R., Massone. G., \& Morbidelli, R. 1988, $A B A$, 203, 388

Worley, C.E. \& Heintz, W.D. 1983, Fourth Catalog of Orbits of Visual Binary Stars, Publ. U.S. Naval Obs., XXIV, Pt. 7

Worley, C.E. \& Douglass G.G. 1970, BAAS, 2, No. 2, 226 